\title{
Effect of pair interactions on transition probabilities between inactive and active states: achieving collective behaviour via pair interactions in social insects
}

Article

Accepted Version

Hayashi, Y., Yuki, M., Kikuchi, T., Tsuji, K. and Sugawara, K. (2015) Effect of pair interactions on transition probabilities between inactive and active states: achieving collective behaviour via pair interactions in social insects. Journal of the Physical Society of Japan, 84 (10). 104801. ISSN 1347-4073 doi: https://doi.org/10.7566/JPSJ.84.104801 Available at https://centaur.reading.ac.uk/40676/

It is advisable to refer to the publisher's version if you intend to cite from the work. See Guidance on citing.

Published version at: http://journals.jps.jp/doi/abs/10.7566/JPSJ.84.104801

To link to this article DOI: http://dx.doi.org/10.7566/JPSJ.84.104801

Publisher: Physical Society of Japan

All outputs in CentAUR are protected by Intellectual Property Rights law, including copyright law. Copyright and IPR is retained by the creators or other copyright holders. Terms and conditions for use of this material are defined in the End User Agreement. 


\section{www.reading.ac.uk/centaur}

\section{CentAUR}

Central Archive at the University of Reading

Reading's research outputs online 


\title{
Effect of pair interactions on transition probabilities between inactive and active states
}

\section{- Achieving collective behaviour via pair interactions in social insects -}

\author{
Yoshikatsu Hayashi $^{1 *}$, Mai Yuki ${ }^{2}$, Tomonori Kikuchi ${ }^{3}$, Kazuki Tsuji $^{4}$, Ken Sugawara ${ }^{2}$ \\ ${ }^{1}$ Brain Embodiment Lab, School of Systems Engineering, University of Reading, P. O. Box \\ 225, Whiteknights, Reading RG6 6AY, United Kingdom \\ ${ }^{2}$ Graduate School of Human Informatics, Tohoku Gakuin University, 2-1-1 Tenjinzawa, \\ Izumi-ku, Sendai, Miyagi 981-3193, Japan \\ ${ }^{3}$ Marine Biosystems Research Center, University of Chiba, 1-10835-6 Sotogawa \\ Choushi-shi, Chiba 288-0014, Japan \\ ${ }^{4}$ Department of Subtropical Agro-Environmental Sciences, University of The Ryukyus, 1 \\ Senbaru, Nishihara-cho, Nakagami-gun, Okinawa, Japan
}

To understand the evolution of well-organized social behaviour, we must first understand the mechanism by which collective behaviour establishes. In this study, the mechanisms of collective behaviour in a colony of social insects were studied in terms of the transition probability between active and inactive states, which is linked to mutual interactions. The active and inactive states of the social insects were statistically extracted from the velocity profiles. From the duration distributions of the two states, we found that 1) the durations of active and inactive states follow an exponential law, and 2) pair interactions increase the transition probability from inactive to active states. The regulation of the transition probability by paired interactions suggests that such interactions control the populations of active and inactive workers in the colony.

KEYWORDS: Social insects, Mutual interactions, Exponent law, Transition probability of states

\section{Introduction}

A well-organized group can arise by two possible mechanisms: central control and distributed control. In the first mechanism, the colony adapts to its changing environment under the control of a central governor who dictates the activities of other colony members. In the second mechanism, the control is distributed via local interactions among interchangeable

${ }^{*}$ E-mail: y.hayashi@ reading.ac.uk 
components of the colony. ${ }^{1)}$ These collective behaviours are not dominated by a special individual that controls the behaviour of the entire group; rather, they emerge when potentially interchangeable individuals interact through direct sight, direct physical contact or chemicals such as pheromones.

Social insects such as ants and bees display interesting characteristics, including wellorganized group behaviours. Colonies are especially well-organized and exhibit a wide variety of remarkable behaviours. ${ }^{2-4)}$ To understand how colony behaviour emerges from the behaviour of individual workers, Cole observed spontaneous bursts of activity in single ants. Cole noted that such activities incite rhythmic activity of the whole colony as the number of nestmates increases, ${ }^{5)}$ supporting the synchronization paradigm developed by Kuramoto. ${ }^{6}$

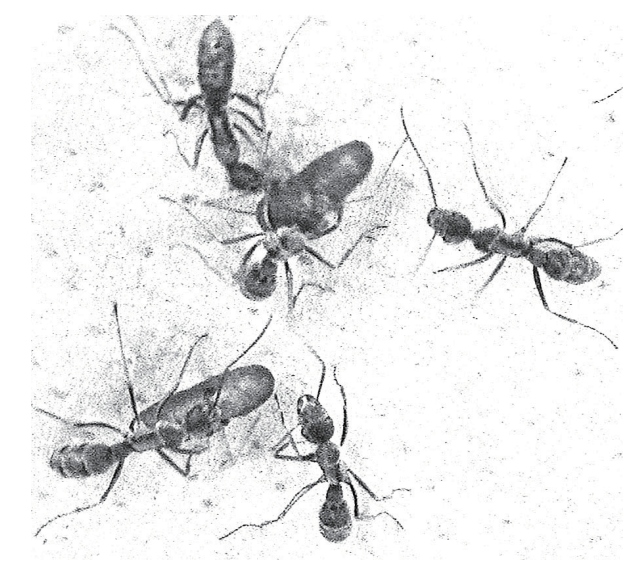

Fig. 1. Observed interactions among workers in an ant colony.

In ant colonies, collective behaviour chiefly relies on local interaction, i.e. direct physical contact between two ants. For illustrative purposes, consider the behaviour in a small group of individuals (Fig. 1). The behavioural pattern reveals the internal states of individual workers and the effect of pair interactions on the transition of those states. By adopting such a bottomup approach, we can understand the interactions among the interchangeable components in social groups, which ultimately lead to collective behaviour.

The behaviour emerging from the dynamics of internal states appears to be universal, and has been reported in fields as diverse as physics, biology and sociology. For example, the scale-invariance property has been reported in the foraging behaviour of Drosophila, ${ }^{8)}$ albatrosses, ${ }^{9)}$ and marine predators. ${ }^{10)}$ Human dynamics models have also discovered a universal law in the cumulative distribution function of active and inactive states. ${ }^{12)}$ The mathematical model of the scale-invariance property assumes that the internal states of the individual 
essentially determine the group behaviour. ${ }^{11)}$

A recent study investigated the ratio of active to inactive workers after a demographic change. ${ }^{7)}$ After the change, some previously inactive workers started to work, whereas some previously active workers became inactive. These shifts restored a substantial amount of variation in the working degree. Therefore, the maintenance of the working population appears to depend on the underlying social mechanism.

Using social insects as a model, the present study aims to link the universal behaviours of individual members with the collective behaviour of the entire colony. To this end, we developed a novel experimental paradigm in an extremely artificial environment, but which allows us to extract the velocity and trajectories of the individual workers under certain experimental conditions. The behavioural patterns were statistically characterized in terms of individual active and inactive states. Primarily, we aspire to reveal the effects of mutual interactions on the active and inactive states of individual colony members.

\section{Methods and Analysis}

\subsection{Materials}

We experimentally investigated the behaviour of the ant genus Diacamma, of which a single species is distributed through Japanese islands of Okinawa. A Diacamma colony comprises 20-300 workers, each approximate length $10 \mathrm{~mm}$ (on average). Workers individually hunt other insects and are aggressive in character. In this species, workers are ranked by their direct physical contacts between individuals. ${ }^{13)}$ The gamergates (functional queens) can inhibit the development of workers' ovaries by direct physical contact, ${ }^{14,15)}$ but only if such contacts are sufficiently frequent.

Our studied colonies were collected from the main island of Okinawa, and were maintained by the standard procedure; that is, in a plastic cage with a moistened plaster floor, as shown in Fig. 2. ${ }^{14)}$ To understand the division of labour among the entire colony, we designated workers selected from the nest as 'internal workers' and those picked from the feeding site as 'external workers' (Fig. 2). The workers of this species display age-polyethism, meaning that most of the internal workers are young workers engaged in nursing and nest maintenance, whereas the external workers mostly comprise older workers that are foragers and nest defenders. ${ }^{16)}$ 


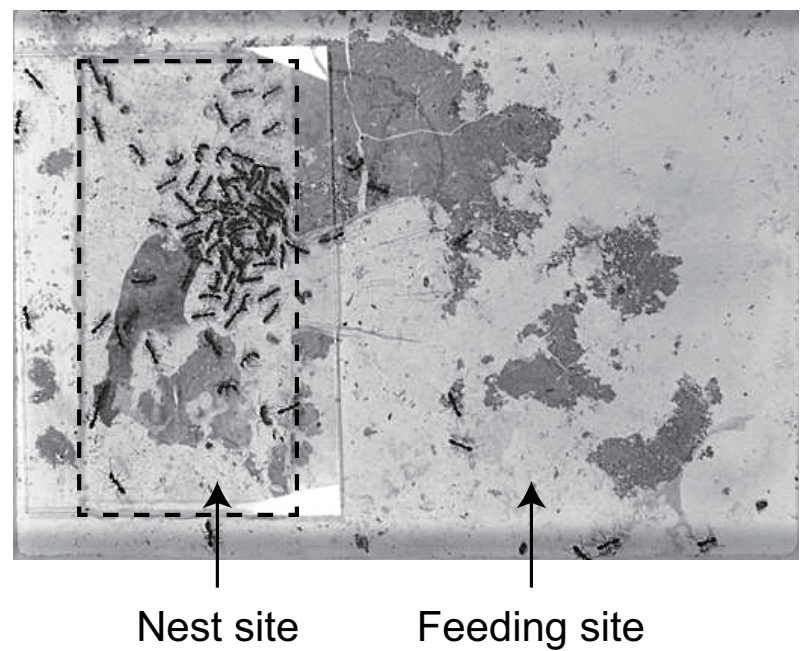

Fig. 2. Rearing environment of the colony. To distinguish among the two worker classes, the internal and external workers were taken from the nest site and the feeding site, respectively. The nest site (covered by the transparent board) is delineated by the dashed line. Food was always placed at the feeding site.

\subsection{Experimental set-up}

To avoid artefacts introduced by the boundaries, the trajectories of the individuals should be distributed throughout the entire space. Previously, we achieved a relatively homogeneous distribution of walking worker trajectories in a hemispherical domain. ${ }^{17)}$ The internal and external workers were randomly picked up from the nest site and feeding site of the rearing environment, respectively, as shown in Fig. 2, and were placed in an acrylic hemispherical field (diameter $=300 \mathrm{~mm}$; see Fig. 3). After the experiments, the pheromone trails left by workers were removed from the acrylic surface by wiping with $75 \%$ ethanol.

Two experimental conditions were imposed in this study; the isolated condition, in which a single worker was placed on the hemisphere, and the paired condition, in which two workers were placed on the hemisphere. The number of internal workers was $N=8$ in the isolated condition, and $N=14$ in the paired condition; for the external workers, we set $N=7$ and $N=14$ in the isolated and paired conditions, respectively. The isolated workers were randomly selected from 8 colonies. Paired workers were randomly chosen nestmates from a colony that was also randomly selected.

\subsection{Measurement and Analysis}

Throughout the experiment, the hemisphere was covered by a transparent acrylic board. The motions of one or two workers across the hemispherical surface were recorded for $6 \mathrm{~h}$ by a CCD camera erected above the hemisphere (Fig. 3). The visual dataset was acquired as 


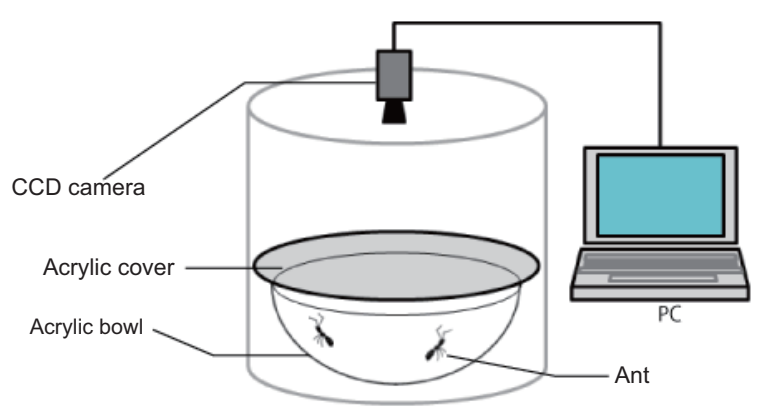

Fig. 3. Schematic of the experimental set-up. The workers were picked up from the rearing environment, and placed on the hemispherical surface. The translational motions of the workers were recorded by the CCD camera positioned above the hemisphere.

a $640 \times 480$ pixel image. The centre of mass of the ant's image was calculated as a function of time, and the trajectories over the hemispherical surface of the hemisphere were projected onto 2D Cartesian coordinates. To correctly obtain the temporal changes in the velocity profiles, we first transformed the Cartesian coordinates of the worker's location to spherical coordinates, so that the velocity reflected the translational motion on the surface of the hemisphere.

The above procedure captures the trajectories of individual workers. To analyse the worker motions under paired conditions, the contact spots of both workers were marked when their centres of area converged to within $50 \mathrm{~mm}$. The spatial distributions of the contact points were recorded across the hemispherical field.

The worker velocities were calculated as functions of time and smoothed by averaging over $60 \mathrm{~s}$. To discuss the statistical properties of the active and inactive worker states, we digitized the temporal pattern, ${ }^{10)}$ i.e. if the velocity of the individual worker exceeded a specified threshold, that worker was considered to be active; otherwise, the worker was inactive. Here, we set the threshold as half of the average velocity of the workers. To extract the statistical properties of each state, we also calculated the durations of the active and inactive states.

From the cumulative duration distributions of the active and inactive states, we extracted the characteristic features of the workers under isolated and paired conditions.

\section{Results and Discussion}

\subsection{Spatial patterns of worker trajectories and worker contact spots}

It is important to confirm that the workers walk across the hemispherical surface in a relatively homogeneous manner and that typical contact behaviour establishes in this artificial environment. Under the paired condition, both workers made physical contact by extending 
their antenna from their bodies, as shown in Fig. 4.

Typical trajectories of two workers under the paired condition are presented in Fig. 5. Both workers covered the entire surface of the hemisphere and made a substantial number of contacts (indicated by black circles in Fig. 5). The contact spots were inhomogeneously distributed on the hemispherical surface and were positively correlated with the local trajectory densities of the two workers.

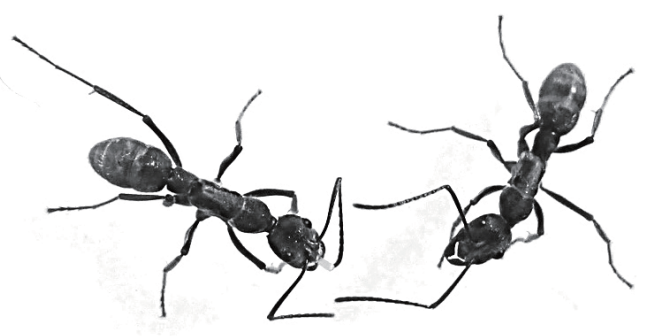

Fig. 4. Snapshot of a paired interaction on the hemispherical surface. The two workers make contact by extending their antennae.

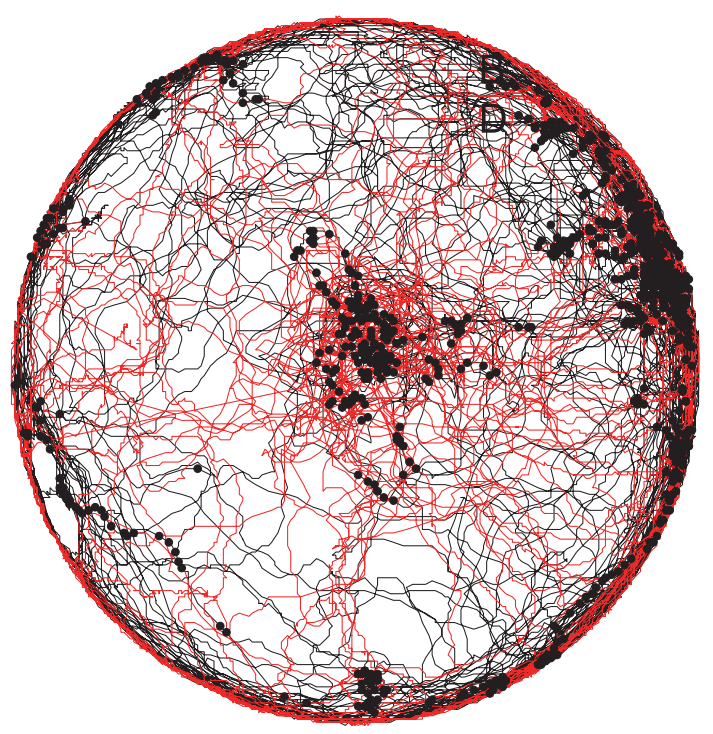

Fig. 5. Typical trajectories of two workers maintained under the paired condition for $6 \mathrm{~h}$. Black and grey lines trace the trajectories of ant $A$ and ant $B$, respectively. Black circles enclose the contact spots of both workers.

Interaction between the two workers under the paired condition was statistically verified. Panels (a) and (b) of Fig. 6 plot the distributions of the distances between two internal workers 
and two external workers, respectively. The histograms peak at approximately $30 \mathrm{~mm}$, indicating that the workers were inclined to make contact rather than wander randomly across the hemisphere.
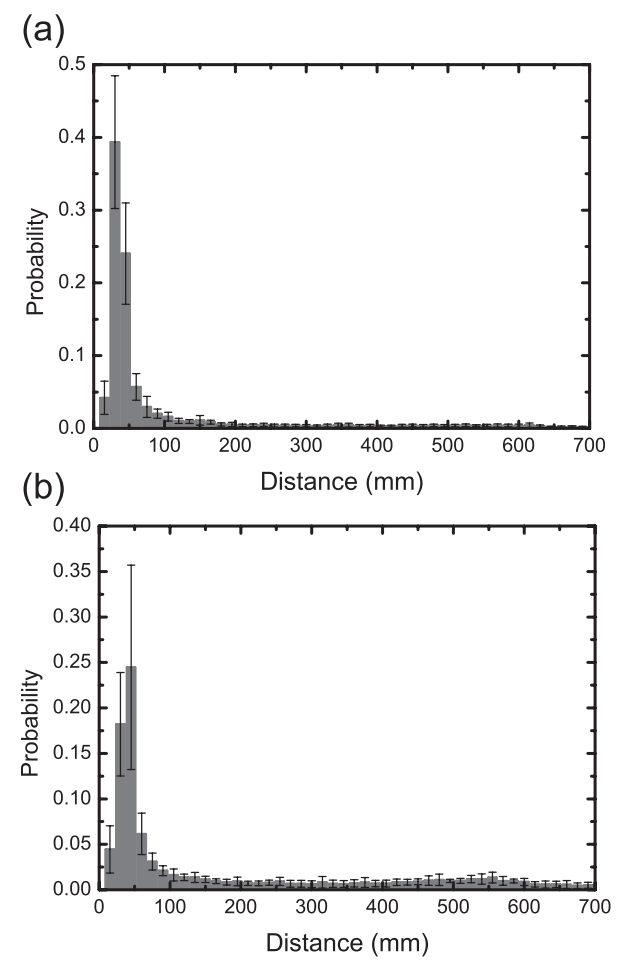

Fig. 6. Distribution of distances between two workers confined on the hemispherical surface; (a) internal workers and (b) external workers. The error bars indicate the standard errors in the means. Distances were sampled at 60-s intervals over $6 \mathrm{~h}$.

\subsection{Temporal patterns of the velocities of the workers}

Typical time development of the velocities of a worker is shown in Fig. 7. To further discuss the statistical properties of the active and inactive states, we must binarize the states. The velocities distributions calculated from Fig. 7 (a) are presented in Fig. 7 (b). The typical velocities of the workers followed a bimodal distribution. The half-average velocity is indicated along the bottom of the bimodal distribution.

As the threshold dividing the active from the inactive states of the workers, we adopted half of the average velocity over the individual time courses of the worker. Although there are some arbitrary elements in the determination of the threshold, it is reasonable to scale the threshold as a function of the average velocity that reflects the overall activity of the individual workers. 
Visual inspection of the workers also indicated that halving the average velocities of individual workers is reasonable from a biological perspective, because it distinguishes between global translational motions and small local motions. Figure 7 (c) shows the active and inactive state occupancies of the workers. From the time courses of the binary states, we calculated the durations of the active and inactive states of the individual workers.

(a)

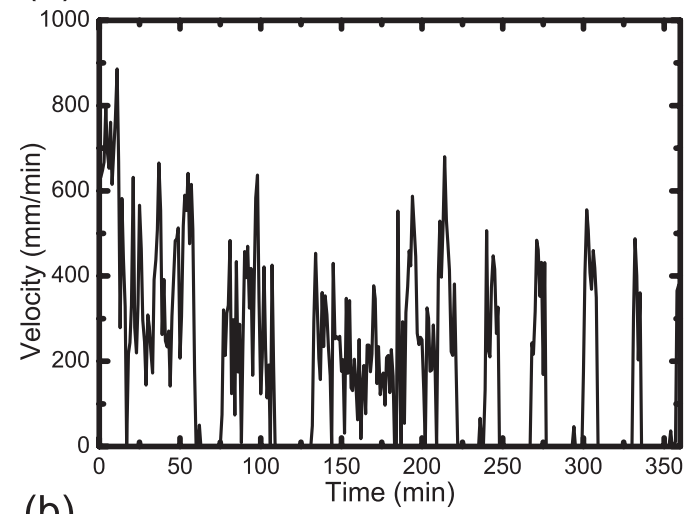

(b)

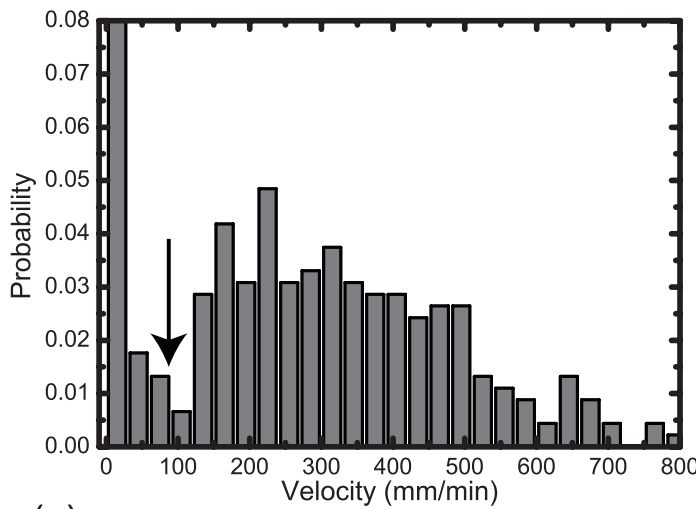

(c)

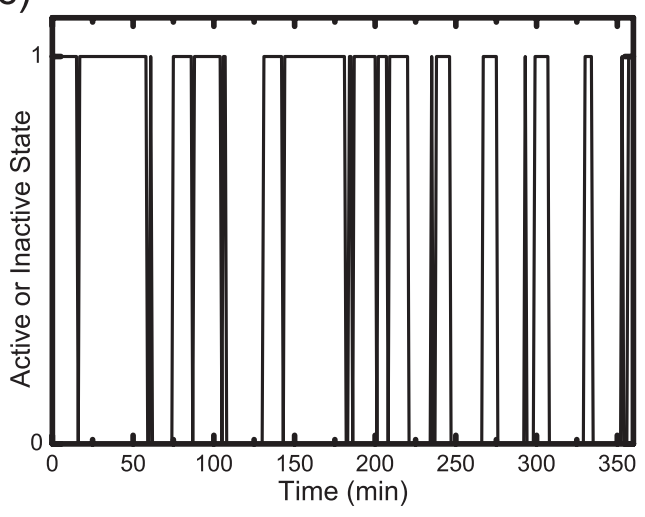

Fig. 7. Temporal patterns of the velocities of a worker. (a) Temporal velocity profiles. (b) Velocity distributions. The half-velocity of the workers, $87.1 \mathrm{~mm} / \mathrm{min}$ is indicated by an arrow. (c) Temporal variations in active (1) and inactive (0) states. 


\subsection{Statistical properties of the active and inactive states}

\subsubsection{Analysis of Internal Worker Behaviour}

Figure 8 presents the average total distances travelled by internal workers under isolated and paired conditions. The total travelling distances of workers in the two groups did not significantly differ (Wilcoxon test; $Z=-0.17, P=0.865$ ).

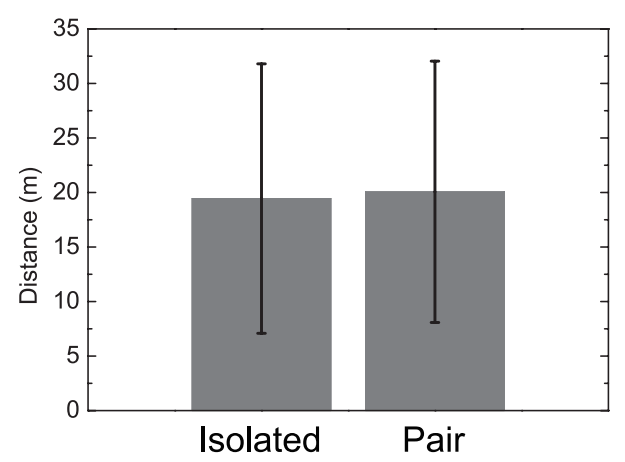

Fig. 8. Average total travelling distances of internal workers under isolated and paired conditions.

Figure 9 plots the cumulative distributions of the times spent in the active and inactive states on a semi-log scale. A linear regression of this plot reveals that the probabilities of existing in the active and inactive states exponentially decrease with state duration. The inactive states of internal workers demonstrate significantly different trends under isolated and paired conditions (Log-rank test, $\chi=64.8, P<0.0001$ ). This difference disappears in the case of external workers (Log-rank test, $\chi=0.127, P<0.722$ ).

The total travelling distances were the same under isolated and pair conditions; only the frequency distributions of the inactive state durations were significantly altered by the pair interactions. Specifically, the exponent of the cumulative frequency distribution was decreased by the pair interactions. The pair interactions exerted no influence on the active state.

\subsection{Analysis of External Worker Behaviour}

Figure 10 presents the average total travelling distances of external workers under isolated and paired conditions. The total travelling distance was not significantly different between single and paired workers (Wilcoxon test, $Z=1.156 P=0.247$ ). However, external workers travelled four times further (on average) than the internal workers throughout the 6-h observation period, reflecting their foraging behaviour.

Figure 11 plots the cumulative distributions of the durations of the active and inactive states on a semi-log scale. Both inactive and active states of the external workers exhibited 


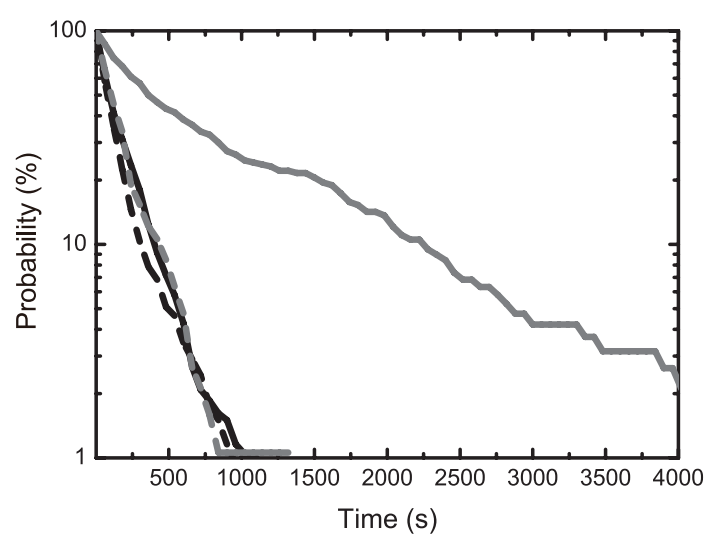

Fig. 9. Cumulative duration distributions of the active and inactive states of internal workers, plotted on a semi-log scale. Black and grey lines denote the pair and isolated conditions, respectively, and the solid and dashed lines denote the inactive and active states, respectively.

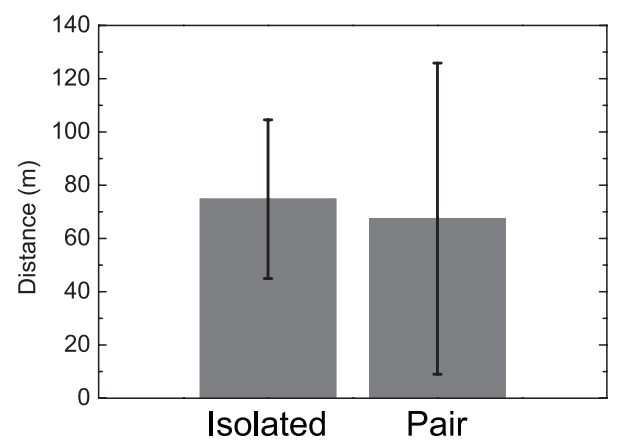

Fig. 10. Average total travelling distances of external workers under isolated and paired conditions.

significantly different trends under isolated and paired conditions (Log-rank test of inactive state: $\chi=4.20, P<0.04$; log-rank test of active state: $\chi=20.17, P<0.0001)$. The slope of this plot was increased by the pair interactions in both states.

\section{General Discussion}

The cumulative distributions of the durations of the active and inactive states were linear functions of the duration on a semi-log scale, indicating an exponential behaviour of state duration (see Figs. 9 and 11). This suggests that the transitions between inactive and active states are describable by a simple transition state theory. Let us assign a transition probability $k_{1}$ from the inactive to the active state and let $k_{2}$ be the probability of the reverse transition. We can now derive the cumulative frequency distributions of the durations $t$ in the active and rest states from the transition probability $k$. If the state randomly changes from inactive to 


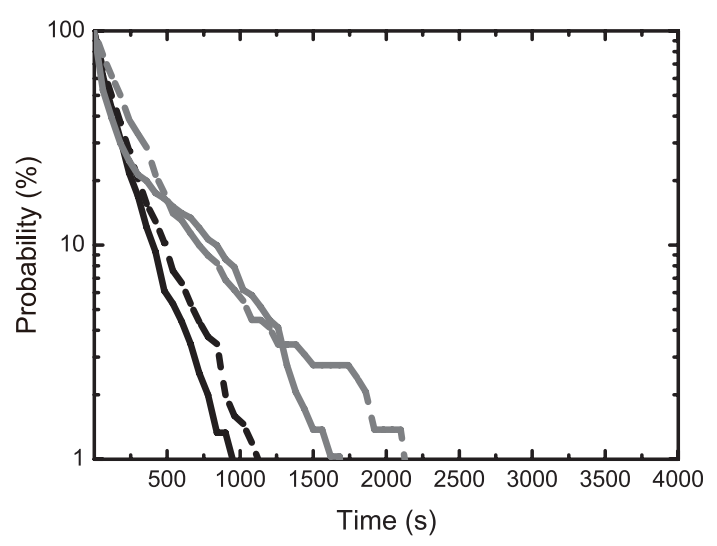

Fig. 11. Cumulative duration distributions of the active and inactive states of external workers, plotted on a semi-log scale. Black and grey lines denote the paired and isolated conditions, respectively, and the solid and dashed lines denote the inactive and active states, respectively.

active between times $t$ and $t+d t$, the transition probability $p_{1}\left(t ; k_{1}\right) d t$ is given by

$$
p_{1}\left(t ; k_{1}\right) d t=\left(1-k_{1} \cdot \frac{t}{n}\right)^{n} \cdot k_{1} \cdot d t
$$

where $n$ is the number of intervals between 0 and $t$. In the limit $n \rightarrow \infty$, we obtain

$$
p_{1}\left(t ; k_{1}\right) d t=k_{1} \exp \left(-k_{1} t\right) d t
$$

Integrating Eq. (2) with respect to time, we obtain an exponential function of the cumulative distributions. We now fit the exponential function [Eq. (2)] to the cumulative frequency distributions plotted in Figs. 9 and 11. The state transition probabilities $k_{1}$ and $k_{2}$ for the internal and external workers are listed in Tables I and II, respectively. The coefficients of determination $R^{2}$ (also listed in Tables I and II) confirm that the cumulative frequency distributions of durations can be described by Eq. (2). Thus, the durations of the active and inactive states follow a Poisson process, and the transition probabilities $k_{1}$ and $k_{2}$ are altered by mutual interactions (Tables I and II).

Interestingly, only $k_{1}$ (the transition probability from inactive to active states) was increased by interactions among pairs of internal workers. This implies that interactions among two internal workers increase the probability of transition from the inactive to active state. On the other hand, interactions between two external workers alter $k_{1}$ and $k_{2}$. We consider that both transition probabilities between the active and inactive states increased via pair interactions, reflecting the statistical significance found in the cumulative duration distributions of the active and inactive states (Fig. 11).

The transition probabilities ratio $k_{2} / k_{1}$ significantly differed only for internal workers un- 
Table I. Exponents of cumulative duration distributions (internal workers; see Fig. 9).

\begin{tabular}{|c|c|c|}
\hline & $k_{1}\left(\times 10^{-3}\right)$ & $k_{2}\left(\times 10^{-3}\right)$ \\
\hline Isolated condition & $1.33\left(R^{2}=0.938\right)$ & $6.21\left(R^{2}=0.994\right)$ \\
\hline Pair condition & $6.38\left(R^{2}=0.992\right)$ & $8.14\left(R^{2}=0.993\right)$ \\
\hline
\end{tabular}

Table II. Exponents of cumulative duration distributions (external workers; see Fig. 11).

\begin{tabular}{|c|c|c|}
\hline & $k_{1}\left(\times 10^{-3}\right)$ & $k_{2}\left(\times 10^{-3}\right)$ \\
\hline Isolated condition & $5.49\left(R^{2}=0.863\right)$ & $3.58\left(R^{2}=0.990\right)$ \\
\hline Pair condition & $6.44\left(R^{2}=0.992\right)$ & $5.33\left(R^{2}=0.993\right)$ \\
\hline
\end{tabular}

der isolated conditions (Fig. 12). Note that when the colony exists in steady state, the ratio $k_{2} / k_{1}$ describes the population ratio between the inactive and active workers within a colony. Therefore, interactions between pairs of colony members should lead to a certain ratio of active and inactive workers within a colony, since the transition probabilities of the states of individual workers are affected by the interactions. Pair interactions can recruit more workers to the active state, thus increasing the total activity of the colony. Only the transition probability ratio $k_{2} / k_{1}$ is expected to be altered by pair interactions between internal workers because internal workers must allocate certain tasks to maintain the whole nest activities. On the other hand, for the external workers, we consider that the transition probability ratio $k_{2} / k_{1}$ does not have to change since they do not have to allocate the tasks inside the colony in which the pair interactions take place.

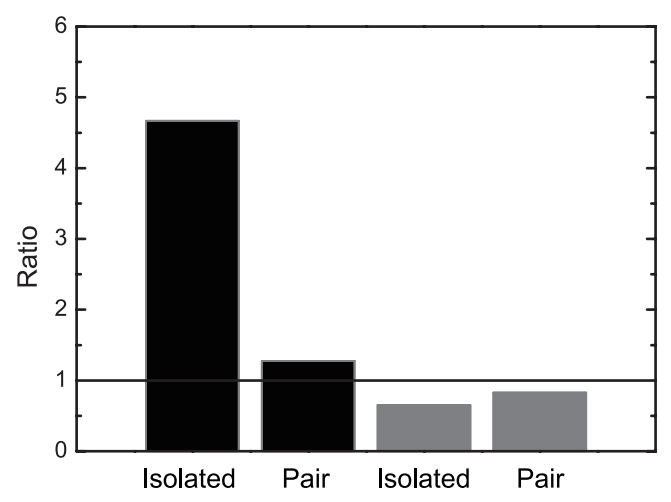

Fig. 12. Ratios of transition probabilities $k_{2} / k_{1}$ for internal workers (black columns) and external workers (grey columns) under isolated and paired conditions. 


\section{Conclusion}

Two main conclusions can be made from this study; 1) both active and inactive states are governed by a Poisson process and 2) the transition probability from inactive to active states of internal workers (but not external workers) is increased by pair interactions, increasing the population of active workers. The derived transition probabilities indicate that if a certain portion of the population is removed, the colony will recover its initial total activity through iterative pair interactions. ${ }^{7)}$ The finding that mutual interactions affect the state transitions of internal workers has implications for the collective behaviours of task allocation. Such interactions provide a fundamental mechanism by which the entire colony engages in collective behaviour. By our bottom-up approach, focusing on isolated and paired individuals, we discovered a simple internal state transition described by the transition probabilities of the Poisson process and the coupling of internal state transitions via mutual interactions.

\section{Acknowledgment}

This study was supported by Grant-in-Aid for Scientific Research on Priority Areas (Mobiligence), No. 18047010, and was partially supported by a Grant-in-Aid for Scientific Research (C) (No. 25330346) of The Ministry of Education, Culture, Sports, Science, and Technology, Japan. 


\section{References}

1) S. N. Beshers and J. H. Fewell, Annu. Rev. Entomol. 46, 413 (2001).

2) B. Hölldobler and E. O. Wilson, The Ants (Harvard University Press, 1990).

3) B. Hölldobler and E. O. Wilson, Journey to the Ants: A Story of Scientific Exploration (Harvard University Press, 1995).

4) D. Gordon, Ants at Work (W. W. Norton Co. Inc., 2000).

5) B. J. Cole, Amer. Nat. 137, 244 (1991).

6) Y. Kuramoto, Chemical Oscillations, Waves, and Turbulence (New York, NY: SpringerVerlag, 1984).

7) Y. Ishii and E. Hasgeawa, J. Ethol, 31-1, 61 (2013).

8) I. Shimada, Y. Minesaki and H. Hara, J. Ethol. 13, 153 (1995).

9) M. G. Viswanathan, V. Afanasyev, V. S. Buldyrev, J. E. Murphy, A. P. Prince and E. H. Stanley, Nature 381, 413 (1996).

10) D. W. Sims, et al., Nature 451, 1098 (2008).

11) I. Shimada, Y. Kawazoe and H. Hara, Biol. Cybern. 68, 477 (1993).

12) T. Nakamura, K. Kiyono, K. Yoshiuchi, R. Nakahara, R. Z. Struzik and Y. Yamamoto, Phys. Rev. Lett. 99, 138103 (2007).

13) H. Shimoji, M.S. Abe, K. Tsuji and N. Masuda, J. R. Soc. Interface 11, 20140599 (2014).

14) K. Tsuji, K. Egashira and B. Holldober, Anim. Behav. 58-2, 337 (1999).

15) T. Kikuchi, T. Nakagawa and K. Tsuji, Anim. Behav. 76, 2069 (2008).

16) K. Nakata, J. Ethol. 13, 113 (1995).

17) Y. Hayashi, M. Yuki, K. Sugawara, T. Kikuchi and K. Tsuji, Robotics and Autonomous Systems, 'Special Issue: Mobiligence' 60, 714 (2012). 